\title{
Fingertip Force Planning During Grasp Is Disrupted by Impaired Sensorimotor Integration in Children With Hemiplegic Cerebral Palsy
}

\author{
ANDREW M. GORDON, JEANNE CHARLES, AND BERT STEENBERGEN \\ Department of Biobehavioral Sciences [A.M.G., J.C.], Teachers College, Columbia University, NY, 10027; NICI, Radboud University \\ Nijmegen, 6500 HE Nijmegen, The Netherlands
}

\begin{abstract}
In the present study we examine the ability of children with hemiplegic cerebral palsy (CP) to use anticipatory control of fingertip forces during grasping, and whether anticipatory control is facilitated by lifts with the contralateral hand. Eight children with $\mathrm{CP}$ (age 4-13) were asked to perform several lifts of either a $250 \mathrm{-g}$ or 500 -g object instrumented with force transducers with one hand, followed immediately by several lifts with the contralateral hand. This was repeated for each combination of weight and starting (involved or non-involved) hand. Similar to previous studies, the rate of load force development showed impaired anticipatory control during lifts with the involved hand, intact anticipatory control in the non-involved hand, and transfer of anticipatory control from the non-involved to the involved hand. Surprisingly, however, we also found a transfer from the involved hand to the non-involved hand. The results suggest that the impaired anticipatory control in the involved hand is not purely a sensory or motor problem, and instead is due to an inability to appropriately integrate sensory information with subsequent motor output of the same hand. These results provide important information about the mechanisms underlying impaired anticipatory control, and may have important clinical implications. (Pediatr Res 60: 587-591, 2006)
\end{abstract}

$\mathrm{D}$ uring object manipulation, the development of isometric fingertip forces must be scaled (planned) before the initiation of the movement since sensory information about the object's weight is not immediately available. This "anticipatory control" is based on prior manipulatory experience with the object (1-3). Interestingly, this anticipatory control can be transferred across effectors in both healthy children and adults since weight (4) and frictional (5) information gained during previous lifts with one hand can be used to scale the fingertip forces during subsequent manipulations with the contralateral hand.

In a series of studies, it has been shown that children with $\mathrm{CP}$ have an impaired ability to plan the force output in advance (6-99). Specifically, children with $\mathrm{CP}$ were unable to

Received March 29, 2006; accepted June 22, 2006.

Correspondence: Andrew M. Gordon, Ph.D., Department of Biobehavioral Sciences, Box 199, Teachers College, Columbia University, 525 West 120th Street, NY, NY 10027; e-mail: ag275@columbia.edu.

Financial support: This project was supported by a grant from the United Cerebral Palsy Research and Education Foundation. The last author was supported by a grant awarded by The Netherlands Organization for Scientific Research (NWO) for the project 'Adaptation in Movement Disorder' (016.005.062).

DOI: $10.1203 / 01$.pdr.0000242370.41469.74 scale the rate of grip and load force increase based on the weight of the object unless provided with sufficient practice with an object (8). Similar to the healthy children and adults, children with $\mathrm{CP}$ were able to transfer the anticipatory control between the two hands; following several lifts with the noninvolved hand, anticipatory control, reflected by force rates that appropriately reflect the object's weight, is immediately present in the involved hand (10). A similar transfer from the non-involved to the involved hand has been observed in adults with hemiplegia (11). Based on this finding, it was hypothesized that the impaired anticipatory control in the involved hand is the result of impaired or indistinct sensory mechanisms signaling the object's physical characteristics $(10,12)$.

If the hypothesis that the impaired anticipatory control is due to disturbed sensory information is correct, then sensory information related to an object's properties from the involved hand would be of little value during subsequent lifts with the non-involved hand. In the present study we investigate this hypothesis by determining whether anticipatory control of fingertip forces is facilitated by lifts with the contralateral involved hand. Specifically, a finding of impaired fingertip force scaling during successive lifts with the involved hand and in the non-involved hand subsequent to lifts with the involved hand would support the hypothesis that the impaired anticipatory control is due to impaired sensation. In contrast, a finding of appropriate fingertip force scaling in the noninvolved hand subsequent to lifts with the involved hand would suggest that the impaired anticipatory control is instead due to in inability to appropriately integrate the sensory information with the motor output of the involved hand (i.e. impaired sensorimotor integration).

\section{METHOD}

Participants. Eight male children with hemiplegic CP (age range 4.6-13.6 $\mathrm{y} / \mathrm{mo}$, mean $8.7 \mathrm{y} / \mathrm{mo}$, SD $3.2 \mathrm{y} / \mathrm{mo}$ ) participated in the present study (Table $1)$. This age range was selected since both anticipatory control of fingertip forcers and transfer between effectors emerges before three years of age in typically developing children (4). The children were recruited from area schools and clinics. Children were chosen who had the ability to grasp and lift a small object between the fingertips of the involved hand, were mainstreamed in the schools and generally had normal cognitive abilities according to the Kaufman Brief Intelligence Test (13), and could follow instructions. Sensory

Abbreviations: BHTT, between-hand transfer-trials; CP, Cerebral palsy; WHAT, within-hand anticipation-trials; 
discriminative capacity of the index finger and thumb was assessed via administration of the two point discrimination task (Table 1) (14). In one participant, the assessment could not be reliably performed as this participant was too young. Informed consent was obtained from all participating children and their parents and the study was approved of by the Teachers College, Columbia University Institutional Review Board.

Apparatus. The grip instrument used allowed measurement of grip (normal) and load (tangential) forces from each contact surface $(4.5 \mathrm{~cm}$ diameter, $4.4 \mathrm{~cm}$ apart) using force transducers (Mini F/T transducer, ATI Industrial Automation, NC; Fx and $\mathrm{Fy}, 0.025 \mathrm{~N}$ resolution) and position using an electromagnetic position-angle sensor (Pohlemus Fastrack, Colchester, VT; $0.075 \mathrm{~mm}$ resolution). The weight of the object could be adjusted to either $250 \mathrm{~g}$ or $500 \mathrm{~g}$ without altering its visual appearance by inserting different masses in the base. The force and position signals were sampled at $400 \mathrm{~Hz}$ and $120 \mathrm{~Hz}$, respectively, using a flexible data acquisition and analysis system (SC/ZOOM, Umeå University, Sweden).

Procedure. Before the experiment, all children washed their hands to remove sweat and excessive oil from the skin. They sat on a chair in front of an adjustable table, adjusted in height to position the forearm approximately horizontal to the table when the object was grasped. The object was aligned with the shoulder of the lifting hand. Children were instructed to grasp the object between the thumb and the index finger ("precision grip") and lift it so that it was adjacent to a marker $5 \mathrm{~cm}$ high. They often used their middle finger against the index finger nail for additional support ("three-digit pinch") and/or lifted the instrument between the thumb and lateral portion of their index finger ("lateral pinch") when using their "involved" hand, which we have shown does not affect the use of anticipatory force scaling (7).

Participants performed a series of 44 lifts of the instrumented object with the involved and the non-involved hand. The lifting sequence is depicted in Table 2. All participants started with the involved hand and the weight of the object was counterbalanced across participants. The order of lifts with the two weights was performed in four basic experimental blocks of 11 lifts. Typically, in an experimental block the object was lifted five times in succession with a specific hand and weight, then shifted by the experimenter to the contralateral side. Five additional lifts were then performed with the contralateral hand before it was shifted back to the first side and lifted one time with the corresponding hand. Each weight occurred twice for each hand in the series of 44 lifts.

Finally, it was established whether weight-related information can be passively transferred, or whether actual lifting of the object was necessary for this transfer. To that aim, seven of the children were asked to place their non-involved hand flat on the table with the palm facing up following the above series of lifts. We then placed the object on their hand for $10 \mathrm{~s}$ while instructing the child not to move it. After this procedure, we removed the object and aligned it with the contralateral, involved hand and asked the child to perform one lift. We then repeated the procedure with the other weight.

Data acquisition and analysis. The grip force rate (dGF/dt) and load force rate $(\mathrm{dLF} / \mathrm{dt})$ were calculated using a \pm 10 point numerical differentiation (calculated within a $\pm 25 \mathrm{~ms}$ window).

As depicted in Table 2, two types of trials were distinguished that were used for subsequent analyses. A within-hand anticipation-trial (Table 2) is every fifth (last in the sequence) trial of the successive lifts of five trials with the same weight and the same hand. This trial reveals the extent to which successive lifting with the same hand leads to anticipatory force control in that same hand. A between-hand transfer-trial (Table 2) is the first trial after transfer of the object to the other hand. This trial tells us the extent information about the object's weight gained by successive lifts of one hand can be used for anticipatory force control in the other hand. For each basic experimental block, 4 trials were used for the analysis (for an example of the first

Table 1. Participant information

\begin{tabular}{lllrc}
\hline Child & $\begin{array}{c}\text { Involved } \\
\text { Side }\end{array}$ & Gender & $\begin{array}{c}\text { Age } \\
\text { (y) }\end{array}$ & $\begin{array}{c}\text { TPD } \\
(\text { I/NI, mm) }\end{array}$ \\
\hline DS & Right & Male & 13.5 & $2 / 4$ \\
LM & Right & Male & 7.3 & $5 / 3$ \\
EM & Right & Male & 13.6 & $5 / 3$ \\
KD & Right & Male & 8.8 & $13 / 3$ \\
JM & Right & Male & 7.8 & $4 / 3$ \\
PP & Left & Male & 6.5 & $3 / 3$ \\
LG & Right & Male & 4.6 & N/A \\
JA & Left & Male & 7.3 & $7 / 3$ \\
\hline
\end{tabular}

TPD, two point discrimination; I, involved hand; NI, non-involved hand (average across the thumb, middle and index fingers).
Table 2. Experimental procedure

\begin{tabular}{clll}
\hline Trial & Involved hand & Non-involved hand & \\
\hline 1 & $500 \mathrm{~g}$ & & \\
2 & $500 \mathrm{~g}$ & & \\
3 & $500 \mathrm{~g}$ & & \\
4 & $500 \mathrm{~g}$ & & \\
5 & $\mathbf{5 0 0} \mathbf{g}$ (WHAT) & $\mathbf{5 0 0}$ (BHTT) & \\
6 & & $500 \mathrm{~g}$ & \\
7 & & $500 \mathrm{~g}$ & \\
8 & & $500 \mathrm{~g}$ & \\
9 & & $\mathbf{5 0 0} \mathbf{g}$ (WHAT) & \\
10 & & & Block II \\
11 & $\mathbf{5 0 0} \mathbf{g}$ (BHTT) & & \\
12 & $250 \mathrm{~g}$ & & \\
13 & $250 \mathrm{~g}$ & & Block III \\
22 & $250 \mathrm{~g}$ & & \\
23 & $500 \mathrm{~g}$ & & \\
24 & $500 \mathrm{~g}$ & & \\
33 & $500 \mathrm{~g}$ & & \\
34 & $250 \mathrm{~g}$ & & \\
35 & $250 \mathrm{~g}$ & & \\
44 & $250 \mathrm{~g}$ & & \\
\hline
\end{tabular}

The four basic experimental blocks are displayed with 11 trials each. All 11 trials are displayed for the first block. The same procedure was used for the subsequent blocks (II-IV).

Weights shown in bold type refer to the trials used for the analysis of within-hand anticipatory force scaling (WHAT) and the between-hand transfer in anticipatory force scaling (BHTT).

basic experimental block, see Table 2), yielding a total of 16 trials per participant. These 16 trials are determined by type of trial (WHAT or BHTT), object weight ( $250 \mathrm{~g}$ or $500 \mathrm{~g}$ ) and hand used (involved or non-involved) yielding 8 unique conditions. In each condition, the values of the two trials were averaged, and these means were used for statistical analysis. We performed both nonparametric (Wilcoxon Sign-Rank) and parametric (repeated measures ANOVA) tests at the $p<0.05$ level. While the results were qualitatively similar independent of the statistical methods used, we report the results of the repeated measures ANOVA. In the first $2 \times 2$ ANOVA (Object weight $[250 \mathrm{~g}, 500 \mathrm{~g}] \times$ Hand used [involved, non-involved]) the mean of the within-hand anticipation-trials were used, to test whether sensory information of object weight can be used for anticipatory force control in the same hand after successive lifts with that hand. The second $2 \times 2$ ANOVA (Object weight $[250 \mathrm{~g}, 500 \mathrm{~g}] \times$ Hand used [involved following non-involved, non-involved following involved]) was performed on the mean of the between-hand transfer-trials, and tests whether weight-related information can be used by the contralateral hand for anticipatory force control. Posthoc tests were performed using Newmann-Keuls procedures.

\section{RESULTS}

Fig. 1 shows grip and load forces traces, their derivatives and vertical position for a representative participant during each of the 8 unique conditions (collapsed for hand used). The load force rates are similar for the two weights during consecutive lifts with the involved hand (Fig. 1A), suggesting absent anticipatory force scaling. In contrast, the load force rate is higher for lifts of the heavier $(500 \mathrm{~g})$ object with the non-involved hand (Fig. 1B). Following transfer from the non-involved hand to the involved hand, the load forced rates are higher for lifts with the heavier weight (Fig. 1C). Likewise, following transitions from the involved hand to the non-involved hand, the load forced rates are also higher for lifts with the heavier weight (Fig. 1D). The grip force rates were not appreciably higher in any of the conditions $(p>0.05$ in all cases). Therefore, only the statistical results for the load force rates are described in detail below. 


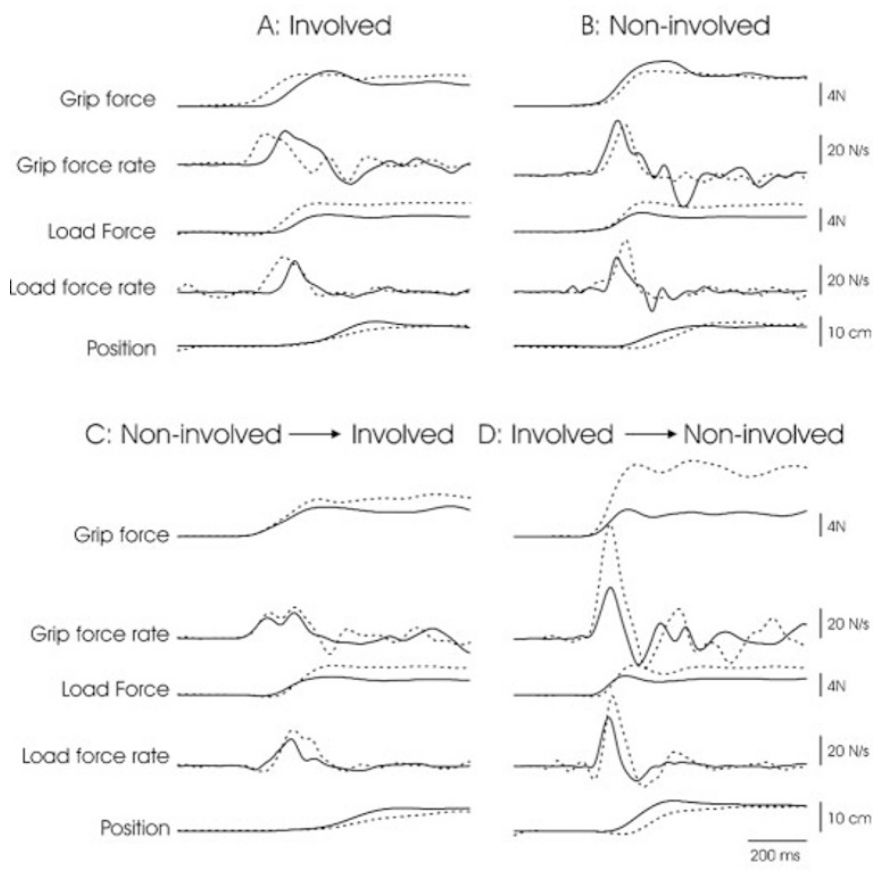

Figure 1. Grip force, load force, their derivatives and position from a representative child with hemiplegia during the fifth consecutive lift using the $(A)$ involved and $(B)$ non-involved hand, and $(C)$ the first lift of the involved hand following five lifts with the non involved hand, and D) the first lift of the non-involved hand following five lifts with the involved hand. Lifts with the 250 -g object (solid traces); lifts with the 500g object (dashed traces). The traces are aligned at the onset of positive load force and the grip and load force rates are shown using a \pm 10 -point numerical differentiation.

Within-hand anticipatory force scaling. As seen in Fig. 2, there were lower load force rates for lifting the 250-g object compared with lifting the $500-$ g object $(24.6 \mathrm{~N} / \mathrm{s}$ and $35.9 \mathrm{~N} / \mathrm{s}$, respectively) when the non-involved hand was used, but not when the involved hand was used $(18.3 \mathrm{~N} / \mathrm{s}$ and $20.2 \mathrm{~N} / \mathrm{s}$, respectively) (main effects of object weight $(F(1,7)=18.3, p$

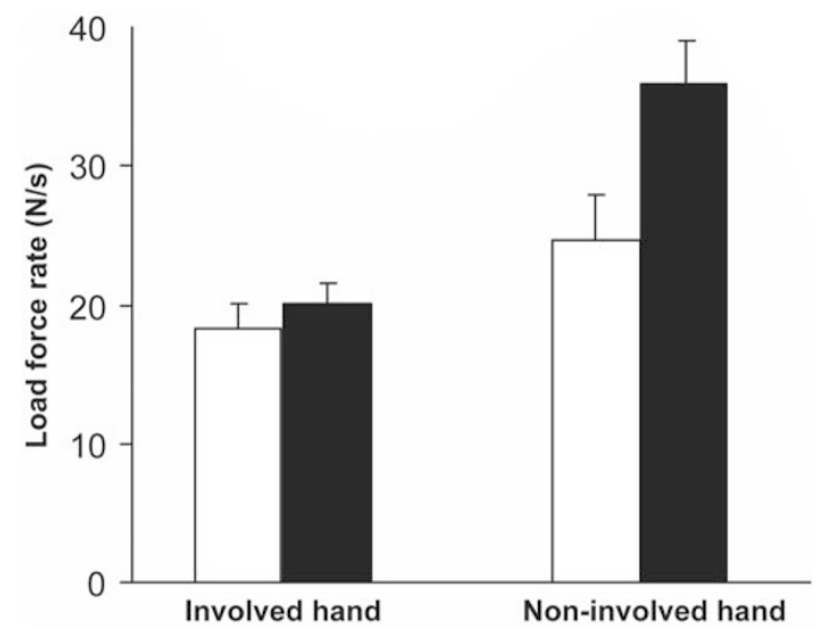

Figure 2. Within-hand anticipation. Means and standard errors across subjects $(n=8)$ of load force rates for the fifth consecutive lift using the involved hand (left bars) and the non-involved hand (right bars). Lifts with the $250-\mathrm{g}$ object $(\square)$; lifts with the 500 -g object $(\square)$. The difference between $250 \mathrm{~g}$ and $500 \mathrm{~g}$ was significant for the non-involved hand (right bars), but not for the involved hand (left bars) (hand $\times$ weight interaction, $p<0.05$ ).
$<0.01$, hand $\times$ weight interaction $((F(1,7)=9.6, p<0.05)$. Thus, the participants demonstrated impaired anticipatory control in the involved hand.

Between-hand transfer in anticipatory force scaling. As seen in Fig. 3, when subjects lifted the object with their involved hand after lifts with the non-involved hand, the load force rates were appropriately scaled to the object's weight $(15.7 \mathrm{~N} / \mathrm{s}$ and $19.6 \mathrm{~N} / \mathrm{s}$, for the $250 \mathrm{~g}$ and $500 \mathrm{~g}$, respectively). Similarly the load force rates were higher when lifting with the non-involved hand following lifts with the involved hand $(23.8 \mathrm{~N} / \mathrm{s}$ and $33.2 \mathrm{~N} / \mathrm{s}$, for the $250 \mathrm{~g}$ and $500 \mathrm{~g}$, respectively (main effect of object weight $(F(1,7)=25.7, p<0.01$, hand $\times$ weight interaction $p>0.05$ ). Thus, anticipatory control could be transferred between the two hands.

Passive transfer. To determine whether an active lift of a given weight is required for generalization of anticipatory control, the children performed one lift with each weight using the involved hand after it was first placed passively in their resting hand. Four participants had higher load force rates forces for the heavier weight, but overall this was not significant ( $t$-test, $p>0.38$ ).

\section{DISCUSSION}

The present findings replicate earlier result in children with hemiplegic $\mathrm{CP}$ of: 1) impaired anticipatory control in the involved hand, 2) intact anticipatory control in the noninvolved hand, and 3) generalization of anticipatory control from the non-involved to the involved hand. The additional finding of a transfer from the involved hand to the noninvolved hand, however, does not support the hypothesis that the impaired anticipatory control in the involved hand is due to indistinct sensory information. Instead, the results suggest that the impaired anticipatory control is due to an inability to appropriately integrate sensory information from the involved

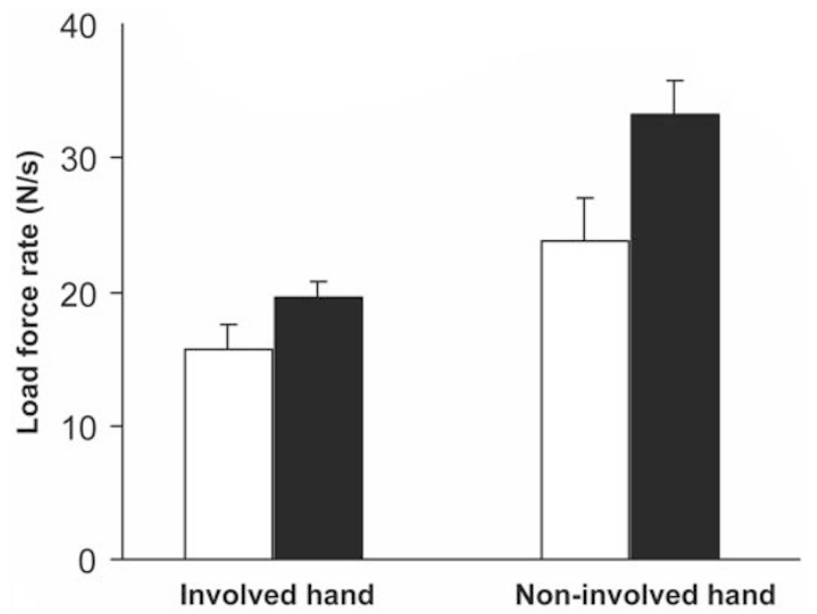

Figure 3. Between-hand transfer. Means and standard errors across subjects $(n=8)$ of load force rates for lifts with the involved hand immediately following five lifts with the non-involved hand (left bars) and the noninvolved hand immediately following five lifts with the involved hand (right bars). Lifts with the 250 -g object $(\square)$; lifts with the 500-g object (ם). For both between-hand transfers, the difference in load force rate between the 250-g object and the 500-g object was significant $(p<0.01)$. 
hand with subsequent motor output of the same hand. These results, and their clinical implications, are described below.

Impairments in anticipatory force control. In agreement with earlier studies, children with hemiplegic CP use a default force scaling in their involved hand, regardless of object weight (6-8), suggesting impaired anticipatory force control. However, with enough practice, children with CP can learn to differentiate the force scaling according to object weight $(7,8)$, indicating that the lack of anticipatory scaling in other studies was not due to an inability to graduate the force output (i.e. an impairment in motor execution).

Anticipatory control of grasping involves activation of cortical regions, including contralateral primary motor cortex, dorsal premotor cortex and ipsilateral posterior parietal cortex $(15,16)$. Ipsilateral posterior parietal may be involved in sensorimotor integration during grasp force coordination (17), whereas premotor cortex may be involved in selection (planning) of motor responses based on learned associations with sensory input formed in the motor cortex (18-20). This importance of the motor cortex in planning is supported by a recent study in which repetitive transcranial magnetic stimulation applied over the motor cortex disrupted anticipatory scaling of fingertip force of the contralateral hand (18). Thus, interruption of motor cortical/corticospinal output in the damaged hemisphere and or in these other cortical regions could account for impaired anticipatory scaling of fingertip forces in the involved hand. Intra-hemispheric connections involving sensory input to motor cortex (21), which are needed to establish sensorimotor associations, may also be damaged, impairing sensorimotor integration needed for anticipatory control in the involved hand.

Based on the finding of an ability to gradually learn to use anticipatory control with extensive practice, we had hypothesized that the initially impaired anticipatory control was due to impaired sensory input (7). This hypothesis was supported by the fact that anticipatory control was immediately present in the involved hand after lifts with the non-involved hand (10). Such transfer of anticipatory control during grasping has been shown in healthy children and adults (4).

The present finding that anticipatory control may be transferred from the involved hand to the non-involved hand despite the fact that it was not present in involved hand is puzzling, and the finding does not support our initial hypothesis that the anticipatory control in the involved hand is due to impaired sensation. If this were the case, it should not be possible to use anticipatory force scaling in either hand following initial lifts with the involved hand. Furthermore, twopoint discrimination was only slightly impaired if at all in most of these children, yet they all had impaired anticipatory control during successive lifts with the involved hand. Instead, the findings suggest that the impaired anticipatory control may be due to an inability to integrate the sensory information with the motor command in the involved hand.

Damage to the corticospinal tract is associated with increased contribution from ipsilesional premotor areas (22-24), which unlike motor cortex, do not have access to ipsilateral primary sensory input (21) needed to form sensorimotor associations. However, we speculate that sensory information of each hemisphere may be transferred to appropriate areas involved in sensorimotor integration in the contralateral cortex via callosal connections. The role of corpus callosum in the transfer of anticipatory control between hands is supported by the finding that the transfer is diminished in a patient with corpus callosum agenesis (4).

The necessity of sensorimotor integration is supported by the results of the trials in which an object was placed passively placed on the subject's non-involved hand before lifting it with their involved hand. These trials show that intact sensory information alone is not enough for anticipatory control, but instead it must be integrated with motor-related information.

The lack of anticipatory scaling of grip forces either within or between hands is in agreement with other studies suggestion that load force rates are more readily scaled within the weights lifted (4). The discrepancy between load and grip force scaling may be further explained by the increased grasping forces in individuals with $\mathrm{CP}(7,10)$, reducing requirement for anticipatory control of grasp force.

Clinical implications. Children with hemiplegic CP also have impaired bimanual coordination above and beyond their unilateral upper extremity impairments (9,25-28). Bimanual coordination impairments may underlie some of the functional limitations these children experience in activities such as dressing, eating, and playing sports. The present results suggest that the non-involved extremity could be used a template for the involved extremity (e.g. kinematic mirroring). Furthermore, it could be used to extract appropriate sensorimotor information about objects manipulated with the non-involved extremity. Together, these may form the basis for intensive bimanual treatment to improve bimanual coordination.

Acknowledgments. We thank the parents and children who made this project possible.

\section{REFERENCES}

1. Johansson RS, Westling G 1988 Coordinated isometric muscle commands adequately and erroneously programmed for the weight during lifting task with precision grip. Exp Brain Res 71:59-71

2. Gordon AM, Westling G, Cole KJ, Johansson RS 1993 Memory representations underlying motor commands used during manipulation of common and novel objects. J Neurophysiol 69:1789-1796

3. Salimi I, Hollender I, Frazier W, Gordon AM 2000 Specificity of internal representations underlying grasping. J Neurophysiol 84:2390-2397

4. Gordon AM, Forssberg H, Iwasaki N 1994 Formation and lateralization of internal representations underlying motor commands during precision grip. Neuropsychologia 32:555-568

5. Johansson RS, Westling G 1984 Roles of glabrous skin receptors and sensorimotor memory in automatic control of precision grip when lifting rougher or more slippery objects. Exp Brain Res 56:550-564

6. Eliasson AC, Gordon AM, Forssberg H 1992 Impaired anticipatory control of isometric forces during grasping by children with cerebral palsy. Dev Med Child Neurol 34:216-225

7. Gordon AM, Duff SV 1999 Fingertip forces during object manipulation in children with hemiplegic cerebral palsy. I: anticipatory scaling. Dev Med Child Neurol 41:166-175

8. Duff SV, Gordon AM 2003 Learning of grasp control in children with hemiplegic cerebral palsy. Dev Med Child Neurol 45:746-757

9. Steenbergen B, Verrel J, Gordon AM In Press Motor planning in Cerebral Palsy. Disabil Rehabil

10. Gordon AM, Charles J, Duff SV 1999 Fingertip forces during object manipulation in 
children with hemiplegic cerebral palsy. II: bilateral coordination. Dev Med Child Neurol 41:176-185

11. Raghavan P, Krakauer JW, Gordon AM 2006 Impaired anticipatory control of fingertip forces in patients with a pure motor or sensorimotor lacunar syndrome. Brain 129:1415-1425

12. Gordon AM, Duff SV 1999 Relation between clinical measures and fine manipulative control in children with hemiplegic cerebral palsy. Dev Med Child Neurol 41:586-591

13. Kaufman AS, Kaufman NL 1990 Kaufman brief intelligence test. American Guidance service, Circle Pines, MN

14. Mackinnon SE, Dellon AL 1985 Two-point discrimination tester. J Hand Surg [Am] 10:906-907

15. Kinoshita H, Oku N, Hashikawa K, Nishimura T 2000 Functional brain areas used for the lifting of objects using a precision grip: a PET study. Brain Res 857:119-130

16. Schmitz C, Jenmalm P, Ehrsson HH, Forssberg H 2005 Brain activity during predictable and unpredictable weight changes when lifting objects. J Neurophysiol 93:1498-1509

17. Ehrsson HH, Fagergren A, Johansson RS, Forssberg H 2003 Evidence for the involvement of the posterior parietal cortex in coordination of fingertip forces for grasp stability in manipulation. J Neurophysiol 90:2978-2986

18. Chouinard PA, Leonard G, Paus T 2005 Role of the primary motor and dorsal premotor cortices in the anticipation of forces during object lifting. J Neurosci 25:2277-2284

19. Koechlin E, Ody C, Kouneiher F 2003 The architecture of cognitive control in the human prefrontal cortex. Science 302:1181-1185
20. Murray EA, Bussey TJ, Wise SP 2000 Role of prefrontal cortex in a network for arbitrary visuomotor mapping. Exp Brain Res 133:114-129

21. Asanuma H, Arissian K 1984 Experiments on functional role of peripheral input to motor cortex during voluntary movements in the monkey. J Neurophysiol 52:212227

22. Johansen-Berg H, Rushworth MF, Bogdanovic MD, Kischka U, Wimalaratna S, Matthews PM 2002 The role of ipsilateral premotor cortex in hand movement after stroke. Proc Natl Acad Sci USA 99:14518-14523

23. Seitz RJ, Hoflich P, Binkofski F, Tellmann L, Herzog H, Freund HJ 1998 Role of the premotor cortex in recovery from middle cerebral artery infarction. Arch Neurol 55:1081-1088

24. Weiller C, Chollet F, Friston KJ, Wise RJ, Frackowiak RS 1992 Functional reorganization of the brain in recovery from striatocapsular infarction in man. Ann Neurol 31:463-472

25. Hung YC, Charles J, Gordon AM 2004 Bimanual coordination during a goaldirected task in children with hemiplegic cerebral palsy. Dev Med Child Neurol 46:746-753

26. Steenbergen B, Hulstijn W, de Vries A, Berger M 1996 Bimanual movement coordination in spastic hemiparesis. Exp Brain Res 110:91-98

27. Steenbergen B, Van Thiel E, Hulstijn W, Meulenbroek RG 2000 The coordination of reaching and grasping in spastic hemiparesis. Hum Mov Sci 19:75-105

28. Gordon AM, Steenbergen B In Press Bimanual coordination in children with cerebral palsy. In: Eliasson AC, Burtner P (eds) Child with Cerebral Palsy: Management of the Upper Extremity. MacKeith Press, London 